\title{
Averaged occipital responses to stimulation of sites in the upper and lower halves of the retina
}

\author{
R. G. EASON, C. T. WHITE, AND D. ODEH 1 \\ SAN DIEGO STATE COLLEGE AND NAVY ELECTRONICS LABORATORY
}

Averaged responses of the occipital lobes to flashes itmposed along the midline of the upper part of the retina are larger and of shorter latency than responses to flashes imposed on the lower retina. Since reaction times also are shorter when the upper retina is stimulated, this study provides further evidence for the generalization that reaction time varies inversely with amplitude and directly with latency of the evoked response.

Several studies have shown that changes in reaction time are accompanied by variations in amplitude and/or latency of evoked cortical potentials (Dustman \& Beck, 1965; Donchin \& Lindsley, 1966; Morrell \& Morrell, 1966). In accordance with these findings we recently observed concomitant changes in reaction time (RT) and evoked potentials (EPS) as the eye was stimulated progressively more peripherally along a horizontal meridian passing through the fovea (Eason, Oden, \& White, 1967). In general, these studies indicate that the amplitude of the EP varies inversely and its latency varies directly with RT.

In comparing the EPs of the right occipital lobe evoked by stimuli impinging upon the nasal retina of the right eye with those evoked by temporal retinal stimulation of the same eye of a left-handed $S$, we encountered a marked exception to the above generalization (Eason \& White, 1967). Surprisingly, EPs to nasal stimulation were of lower magnitude than those evoked by temporal stimulation despite the well established fact that shorter RTs are obtained to stimuli impinging on the nasal retina (Poffenberger, 1912; Woodworth, 1938; Rains, 1963).

Recently we obtained some evidence that the deviant result may be due to the fact that the temporal retina of the right eye projects to the right occipital area, from which we were recording, whereas the nasal retina does not. Recording simultaneously from both occipital lobes we found that the lobe receiving primary projections from the retinal area stimulated elicits larger EPs than does the other lobe and, further, that the relative magnitude of the EPs obtained from the two lobes is related to lateral dominance, as manifested by handedness (Eason, Groves, White, \& Oden, 1967).

The present study compares concomitant changes in RT and EPs in relation to stimulation of the upper and lower parts of the retina along the midline. Since the retinal elements located along the midline project to both hemispheres, any differences in EP latency and amplitude to stimulation of homologous sites of the upper and lower parts of the retina may not be attributed to differences in the primary projections to the occipital areas. Since RTs to stimulation of the upper half of the retina are shorter than those to stimulation of the lower half (Woodworth, 1938; Rains, 1963) we would expect EPs of larger amplitude and shorter latency to occur upon stimulation of the upper half. Such a finding would be consistent with the generalization that RT varies inversely with the amplitude and directly with the latency of EPs. In addition, it would provide further indirect evidence that the results of the nasal-temporal study, which were at variance with this generalization, were due to the projection of the two retinal halves to opposite hemispheres.

\section{METHOD}

Each of the authors served as Ss in two experimental sessions. One of them (RGE) was the lefthanded $S$ in the nasal-temporal study. Retinal sites located 10 degrees apart along a vertical meridian bisecting the fovea were stimulated with blue light in one of the sessions and with red in the other. Both eyes were stimulated simultaneously. Each $S$ participated in five to seven trials per session, depending on the number of pairs of symmetrical sites stimulated. For RGE, seven pairs of sites located 10 to 70 degrees above and below the fovea were stimulated, the farthermost one being 60 degrees out. Depending on the $S$, a total of 100 or 200 flashes was presented to each site per trial. The flashes were presented at a rate of one per second. Order of stimulation of any pair of sites was balanced withtn a trial by presenting flashes in blocks of 25 to each member of the pair, using an ABABBABA design. Red flashes were presented by passing the light through a No. 26 Kodak Wratten filter; blue ones by passing the light through a No. 48A fllter. The $S$ maintained steady fixation during the presentation of each block of flashes, his head resting on a chin bar.

Evoked potentials were recorded by attaching "active" electrodes to the scalp one inch above the inion and one inch to the right and left of the midline. Reference electrodes were attached to the right and left ear lobes, respectively. The responses were amplified and recorded simultaneously from both lobes with an Offner Type $\mathbf{R}$ dynograph and Mnemotron Computer of Average Transients (CAT). Permanent 

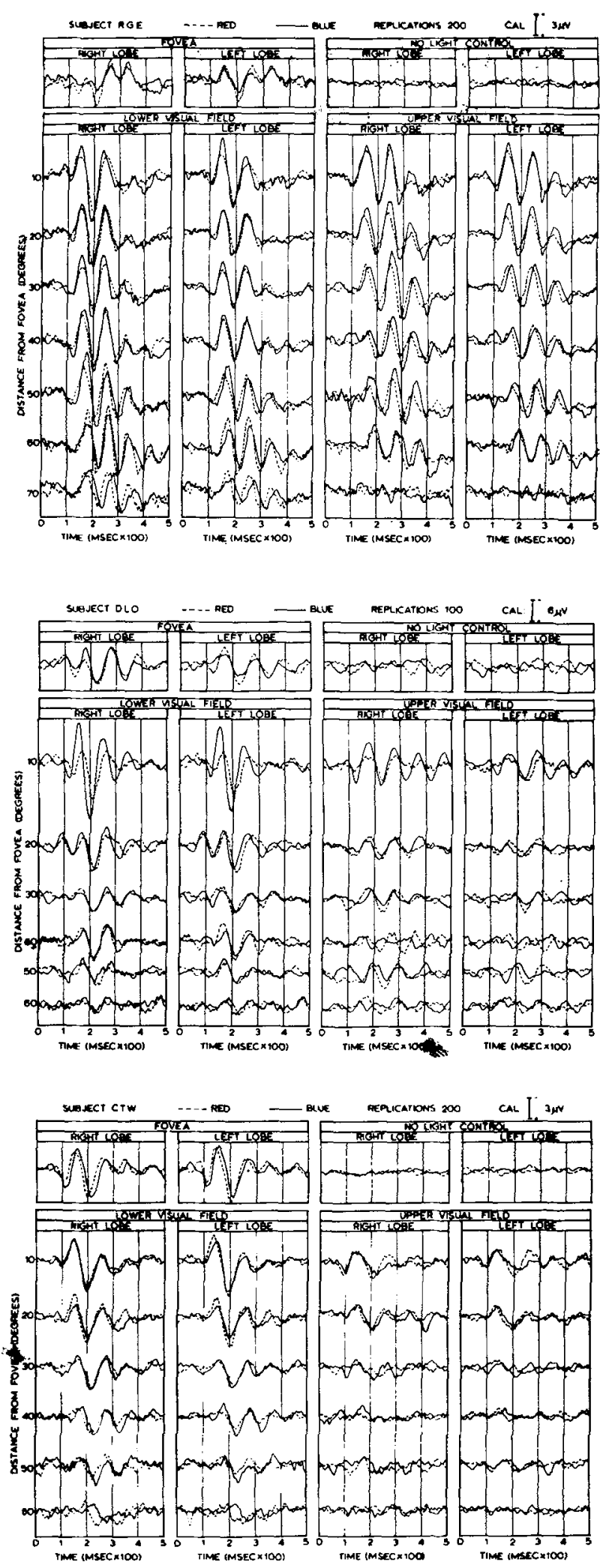

Fig. 1. Evoked responses of right and left occipital lobes to flashes appearing along midline in upper and lower fields at various distances from foveal fixation point. Tracings are categorized by Ss, visual field, lobes, stimulus color, and distance of stimulation site from fovea. records were obtained with a Moseley X-Y plotter. The responses shown in FIg. 1 were reproduced from the $\mathrm{X}-\mathrm{Y}$ plots.

\section{RESULTS AND DISCUSSION}

The evoked potentials obtained in this study are typical of those evoked by a small spot of light, consisting of 8-12 cps sinusoidal deflections which successively increase to a peak amplitude then attenuate (Eason, Oden, \& White, 1967). The first deflection, which is most easily produced upon stimulation of the forea and sites near it, is a low-amplitude, positive excursion (D1) with a latency of about $90 \mathrm{msec}$. The next four or five deflections (D2, D3, etc) constitute the major components of the response. These physical characteristics are evident in the tracings of F1g. 1, wherein the responses of the right and left lobes of each $S$ are categorized with respect to visual field, retinal site, and stimulus color.

In examining the figure it is important to remember that the lower visual field corresponds to the upper half of the retina and the upper field to the lower half. Effect of visual field on the evoked response can be seen by comparing respective lobes, retinal sites, and stimulus colors. A total of 28 such comparisons can be made for subject RGE and 24 for the other two Ss. The effect of stimulating the retina at progressively greater distances from the forea within a given visual field can be observed by scanning each column, whereas effects of color can be ascertalned by comparing each pair of superimposed tracings.

\section{Visual Field Effects}

It is evident upon comparing the tracings of Column 1 to respective tracings of Column 3 that greater responses were obtaited from the right lobe to stimuli appearing in the lower visual field. The same result can be seen for the left lobe upon comparing Columns 2 and 4. A quantitative analysis of the differences attributable to visual fields was made by averaging the amplitude of deflectlons D3, 4, and 5 of each tracing, and counting the number of times that the amplitudes of the responses for respective lobes, colors, and retinal sites were greater for the lower visual field condition. A chi square analysis of each S's data revealed that greater responses occurred slgnificantly more often under the lower visual field condition. A similar analysis of the latency of D4, the large negative deflection beginning at 190-240 msec, revealed that responses of shorter latency occurred stgniflcantly more often for subjects RGE and DLO under the lower visual field condition. All chi squares were stgnificant at the 0.01 level of confidence. Due to the extremely low amplitude of the responses of CTW to stimulation of the retina beyond 20 degrees in the upper field (see Columns 3 and 4 for this S), we were unable to obtain reliable latency measures of D4, and therefore could not ascertain whether latency differences exist in his data. 
The finding that EPs of larger magnitude and shorter latency occur upon stimulation of the upper retina, when considered in conjunction with the fact that reaction times are also shorter, lends further support to the generalization that $R T$ varies inversely with EP amplitude and directly with EP latency. Also, indirect support is provided for the hypothesis that failure to find such relationships upon stimulation of the nasal and temporal halves of the retina is due to the projection of the two retinal halves to different cerebral hemispheres.

Even though RT and EP amplitude and latency are related there is no reason to assume that one has a causal effect on the other. Morrell and Morrell (1966) have delineated several factors known to affect both RT and EP amplitude and/or latency. Subject variables include level of general activation or alertness and focus of attention. Stimulus variables include stimulus intensity, time on task, temporal pattern of stimulus presentation, and others. Concomitant variations in RT and EPs with respect to visual field and distance of the stimulation site from the fovea are correlated with the distribution of rods and cones ( $\varnothing$ sterberg, 1935; Rains, 1963; Payne, 1966, 1967).

\section{Effects of Color and Distance from Fovea}

An examination of the tracings with respect to color reveals marked similarities and few differences. Only the latency of the major components of RGE's responses differed significantly which, on the average, were 8.5 msec shorter for red than for blue. The latency differences became greater as the eye was stimulated progressively more peripherally. With respect to amplitude, a marked difference occurred for DLO at the 10-degree position, the response to blue being greater, but in all other cases the amplitudes were about equal. Even though the changes with respect to color were minor, for the most part, under the conditions of this experiment, it should be pointed out that under certain conditions rather marked differences may be observed (Shipley, Jones, \& Fry, 1965; White \& Eason, 1966).
As the eye was stimulated progressively more peripherally in the upper and lower flelds, responses to both red and blue flashes decreased in amplitude and increased in latency. These variations correspond closely to those obtained when the eye is stimulated progressively more peripherally along the horizontal meridian (Eason, Oden, \& White, 1967).

\section{References}

Donchin, E., \& Lindsley, D. B. Averaged evoked potentials and reaction times to visual stimuli. EEG clin. Neurophysiol., 1966. 20, 217-223.

Dustman, R. E., \& Beck, E. C. Phase of alpha brain waves, reaction time, and visually evoked potentials. EEG clin. Neurophysiol., 1965, 18, 433-440.

Eason, R. G., Groves, P., White, C. T., \& Oden, D. Evoked cortical potentials: Relation to visual field and handedness. Science, 1967, 156, 1643-1646.

Eason, R. G., Oden, D., \& White, C. T. Visually evoked cortical potentials and reaction time in relation to site of retinal stimulation. EEG clin. Neurophysiol., 1967, 22, 313-324.

Eason, R. G., \& White, C. T. Averaged occipital responses to stimulation of sites in the nasal and temporal halves of the retina. Psychon. Sci., 1967, 7, 309-310.

Morrell, L. K., \& Morrell, F. Evoked potentials and reaction times: a study of intra-individual variability. EEG clin. Neurophysiol., $1966,20,567-575$.

Osterberg, G. A. Topography of the layer of rods and cones in the human retina. Acta ophthal. (Kbh.), 1935, Suppl. VI, 102.

Payne, W. H. Reaction time as a function of retinal location. Vision Res., 1966, 6, 729-732.

Payne, W. H. Visual reaction times on a circle about the fovea. Science, 1967, 155, 481-482.

Poffenberger, A. T. Reaction time to retinal stimulation with special reference to the time lost in conduction through nerve centers. Arch. Psychol., 1912, 3, 1-73.

Rains, J. D. Signal luminance and position effects in human reaction time. Vision Res., 1963, 3, 239-251.

Shipley, T., Jones, R. W., \& Fry, A. Evoked visual potentials and human color vision. Science, 1965, 148, 1359-1361.

Woodworth, R. S. Experimental psychology. New York: Holt, 1938. Pp. 327-328.

\section{Notes}

1. D. Oden is now at University of Pennsylvania.

2. This work was supported in part by NSF grant GB-4067.

3. The statements contained herein are the private ones of the authors, and should not be construed ad representing the views of the Navy Electronics Laboratory or the Navy at large.

(Accepted for publication June 26,1967 .) 